\title{
Zur Geschichte der Dermatologie Dessaus
} und seiner Hautklinik

\section{History of Dermatology in Dessau and its Department of Dermatology}

\section{Zusammenfassung}

Es wird hier versucht, die Entwicklung der Dessauer Dermatologie und Hautklinik darzustellen, die sich aus den primitivsten, kriegsbedingten Anfängen zu einer der modernsten Kliniken in Deutschland wandeln konnte. Dies war und ist die Lebensleistung mehrerer Generationen und der Chefärzte Dr. Hermann Ress, Dr. Joachim Theune und insbesondere von Prof. Dr. HansDieter Göring. Ihren Anfang nahm jedoch die Dermatologie in Dessau mit Dr. Fritz Callomon, der am 22. Mai 2006130 Jahre alt geworden wäre und dessen mit diesem Beitrag besonders gedacht sei.

\section{Abstract}

This is an effort to present the history of the Dessauer Dermatology and the development of the Department of Dermatology, which have changed from a most primitive, war-conditioned start to one of the most modern medical complexes in Germany. This was and is the life performance of several generations and of the senior consultants Dr. Hermann Ress, Dr. Joachim Theune and particularly of Prof. Dr. Hans-Dieter Göring. Dermatology in Dessau started, however, with Dr. Fritz Callomon, a practicing dermatologist, who would have been 130 year-old on May 22, 2006 and who is particularly memorized with this contribution.

\section{Einleitung}

Die Geschichte der Hautklinik Dessau ist eng mit dem Schicksal der Stadt Dessau verbunden, wobei das Krankenhauswesen in Anhalts Hauptstadt im 18. Jahrhundert begann. Von 1766 bis 1770 wurde am Leipziger Tor ein „Zucht- und Armenkrankenhaus“ erbaut und eingerichtet. Alle Kranken waren - wie damals üblich - in einem Raum untergebracht. 100 Jahre später wurde 1872 das Kreiskrankenhaus in der Kühnauer Straße 24 eröffnet, dem 1887 ein Erweiterungsbau folgte (Abb.1). Seit dem 1. Mai 1935 gab es die Städtischen Krankenanstalten, in denen auch eine Abteilung für weibliche Prostituierte mit 18 Betten und eine Abteilung für Krätzekranke mit 4 Betten existierte.

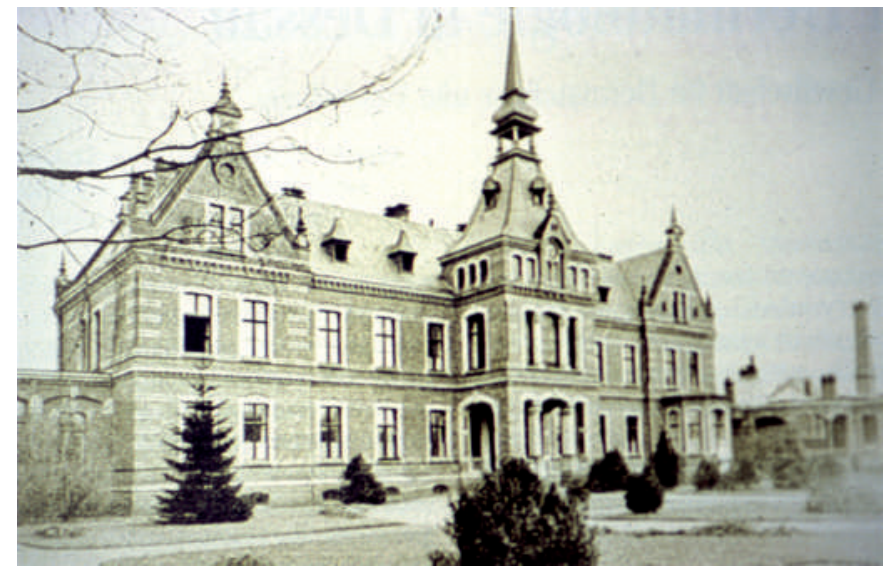

Abb. 1 Altes Kreiskrankenhaus von 1887. 


\section{Dr. Fritz Callomon}

Der erste niedergelassene Dermatologe in Dessau war der jüdische Hautarzt Dr. Fritz Callomon, der 1876 in Breslau geboren wurde, dort studierte und mit „summa cum laude“ bei dem Pädiater Adalbert Czerny zum Dr. med. promoviert wurde. Seine dermatologische Ausbildung erhielt er bei Albert Neisser, dem Entdecker des Gonococcus.

1908 ließ sich Dr. Callomon als Hautarzt im westpreußischen Bromberg nieder. Im 1. Weltkrieg war er Leitender Arzt für Hautkranke im Reservelazarett in Bromberg. 1920, als Westpreußen durch den Versailler Vertrag an Polen gefallen war, verließ Dr. Callomon Bromberg und ließ sich in Dessau als erster Hautarzt in der Albrechtstraße 21 nieder. Dessau hatte damals 75000 Einwohner.

1933 zog er in die Antoinettenstraße 34 um. In der Nummer 24 praktizierte seit 1925 Dr. Hermann Ress, so dass zu dieser Zeit Dessau zwei Hautärzte hatte. Gleich nach der Machtübernahme der Nationalsozialisten kam es zu dem staatlich sanktionierten Terror gegen Juden. Der so genannte „Judenboykott“ vom 1. April 1933 richtete sich gegen jüdische Geschäfte, Rechtsanwälte und Arztpraxen und traf nun auch Dr. Callomon, der 1938 seine Praxis aufgeben musste, da allen jüdischen Ärzten die Approbation entzogen worden war. Er ging mit seiner Frau Dita nach Berlin, wo er untertauchte. Ab 17. August 1938 wurde in alle Reisepässe jüdischer Inhaber ein rotes oder schwarzes großes „J“ eingestempelt und der Inhaber erhielt einen zweiten Zwangsvornamen Israel oder Sarah.

1940 gelang Dr. Callomon endlich die Ausreise nach Amerika, wo er sich noch 64(!)-jährig in Philadelphia/USA eine neue Existenz aufbauen musste. Diese erzwungene Vita erfüllt uns heute noch mit Scham und Zorn.

Im 1. Weltkrieg diente Dr. Callomon seinem Vaterland als Sanitätsoffizier und schrieb 1917 einen richtungsweisenden Artikel über „Hauttuberkulose und Hauttuberkulide bei Heeresangehörigen“, verließ Bromberg, da er als Deutscher nicht unter polnischer Herrschaft leben wollte und musste sich 1940 „davonschleichen“, um das Leben seiner Frau und das eigene zu retten. Mit 64 Jahren in fremder Umgebung, fremder Sprache musste Dr. Callomon nochmals von vorn beginnen, eine Herausforderung, die er jedoch glänzend meisterte. Dr. Callomon verfasste ca. 65 wissenschaftliche Arbeiten, insbesondere ist sein berühmt gewordenes Buch „Die nichtvenerischen Genitalerkrankungen“ zu erwähnen, das 1924 beim Georg Thieme Verlag, Leipzig erschien und mehrere Auflagen, auch im Ausland, erlebte.

Nach dem Krieg hat dann Dr. Callomon auf Bitten Prof. Alfred Marchioninis, Ordinarius für Dermatologie in München und Herausgeber von „Der Hautarzt“, jahrelang die Rubrik „Erlebte Geschichte der Dermatologie“ für den „Hautarzt“ geschrieben.

Dr. Callomon wurde 88 Jahre alt und starb am 15.9.1964 an einem Schlaganfall in San Rafael/Kalifornien.

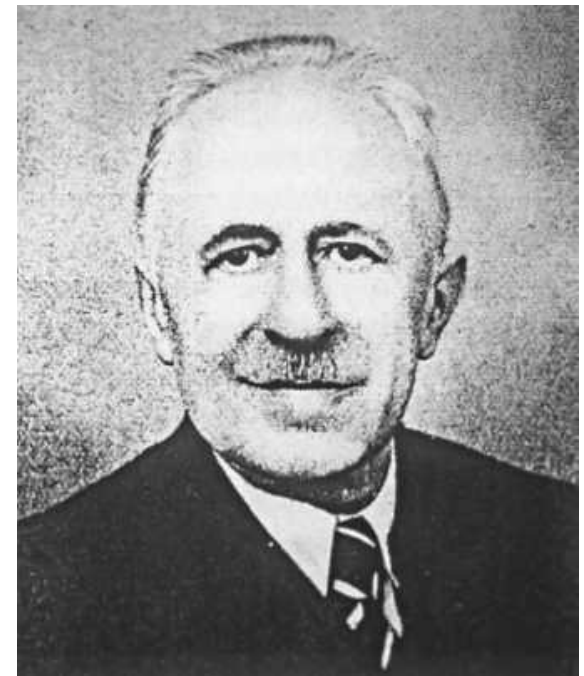

Abb. 2

Dr. Fritz Callomon (1876-1964).

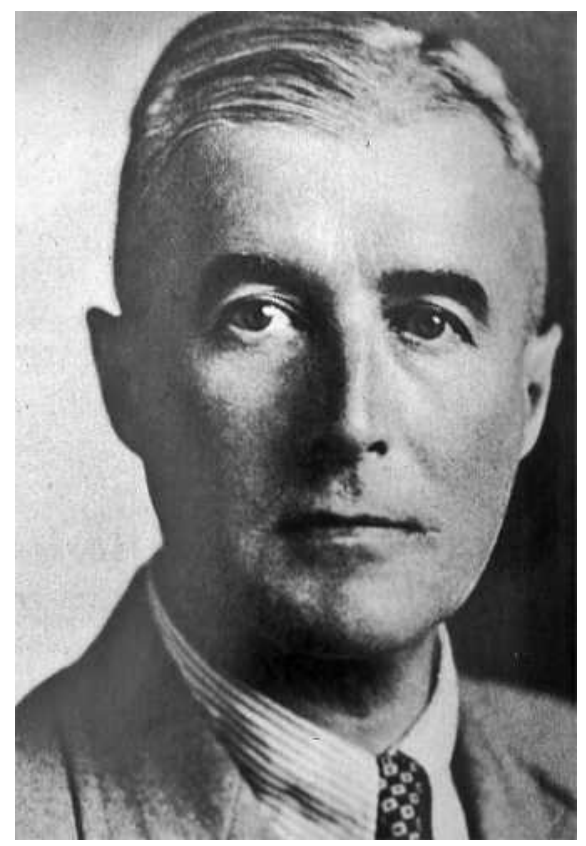

Abb. 3

Dr. Hermann Ress (1891-1975).

\section{Dr. Hermann Ress}

Im 2. Weltkrieg kam es 1942 in Dessau zur Einrichtung von zwei Baracken für Hautkranke, die von einem Assistenten der Inneren Abteilung betreut wurden. Am 15. Juni 1943 wurde in der Gaststätte „Hohenzollernpark“ im Stadtteil Ziebigk eine 40-BettenAbteilung geschaffen, deren Leitung Dr. Hermann Ress (Abb. 3) übernahm. Damit wurden in Dessau erstmalig Hautkranke im stationären Bereich von einem Facharzt betreut. Dr. Ress war gebürtiger Straßburger, Jahrgang 1891.

Die beiden Baracken und die Abteilung „Hohenzollernpark“ brannten bei dem Großangriff auf Dessau vollständig ab. Insgesamt musste Dessau 20 Luftangriffe erdulden, den ersten am 20. August 1940, den schwersten am 7. März 1945 und den letzten am 7. April 1945. Am 7. April 1945 warfen 500 LancasterBomber 1693 Tonnen Bomben ab, die die Stadt zu 85\% zerstörte. 1206 Menschen, standesamtlich registriert, darunter auch viele Kriegsgefangene, Zwangsarbeiter und abgeschossene Bomberpi- 
loten starben kurz vor Kriegsende einen sinnlosen Tod. Auch das Gelände des damaligen Kreiskrankenhauses in der Kühnauer Straße wurde mit seinen 17 Gebäuden dem Erdboden gleich gemacht. 33 Kranke fanden hier den Tod.

Die Haut- und Geschlechtskranken wurden danach in der Hindenburg-Kaserne untergebracht. Nach tagelangen Tieffliegerangriffen zog endlich am 21. April 1945 die 3. US-Panzerdivision in das fast völlig zerstörte Dessau ein. Die Amerikaner requirierten sofort die mit Patienten belegte Hindenburg-Kaserne für sich und setzten die Kranken buchstäblich an die Luft. Prof. Dr. Franz Seeber, der langjährige Ärztliche Direktor des späteren Bezirkskrankenhauses schrieb für die Chronik der Stadt: „Bei ihrem Dienstantritt am 8. Mai 1945 stehen die neu eingesetzten Chefärzte vor dem großen Trümmerfeld der ehemaligen Krankenanstalten in der Kühnauer Straße, das ihnen als Dienststelle übergeben wird. Von den über 800 Betten, auf die das Krankenhaus 1944 angewachsen war, sind kaum noch 100, inklusive der Holzpritschen in dem primitiven Operationsbunker, belegungsfähig.“ Am 3. Juli 1945 erfolgte die Übergabe der Stadt durch die Amerikaner an die Rote Armee. Bereits im gleichen Monat fand die Abteilung für Hautkranke Unterschlupf in der Inneren Abteilung in Dessau-Alten. Anfang 1946 wurden wieder zwei Baracken, diesmal auf dem Gelände der Fliegertechnischen Vorschule in Alten, eingerichtet. Offiziell hatte jede Baracke 50 Betten. Sie waren aber mit 126 Betten belegt. Die Baracke für die Geschlechtskranken war vergittert.

1946 war dort Dr. Gerhard Schmidt, der spätere Stadtrat für Gesundheitswesen, als Pflichtassistent tätig. Von 1946 bis 1950 machte hier Dr. Hans Kleiner, später Dermatologe in Wittenberg, seine Fachausbildung.

Die durchschnittliche Belegungszeit für Hautkranke betrug 1946 19,3 und 1947 24,5 Tage. Die Kosten pro Belegtag beliefen sich 1947 in der 2. Klasse auf 9 Reichsmark und 7 Reichsmark in der 3. Klasse. 1947 wurden in der Ambulanz pro Quartal 2000-2700 Scheine abgerechnet. 1950 stellte der Amtsarzt Dr. Kann vor der Dessauer Stadtverordnetenversammlung fest: „Völlig unzulänglich und jeder Beschreibung spottend sind die Verhältnisse auf der Haut und Geschlechtskrankheiten-Abteilung... Herr Peter vom Landesgesundheitsamt sagte mir, er sei nach meinen Schilderungen auf allerlei gefasst gewesen, doch übertreffe das, was er hier sehe, seine schlechtesten Erwartungen.“ Dr. Kann plädierte für einen Krankenhausneubau in Dessau-Haideburg, der aber nie erfolgte, da er 25 Millionen Reichsmark gekostet hätte, eine damals nicht aufzubringende Summe.

Dr. Ress beklagte 1948 die zunehmende Gonokokkenresistenz durch Sulfonamidtherapie, wodurch sich die Verweildauer mehr und mehr verlängerte. Doch in den Folgejahren sank die Zahl venerischer Neuerkrankungen durch:

- Einführung des Penicillins;

- rigorose Behandlungsmethoden (Provokationen mit Milchoder Terpentinölinjektionen, von den Patienten „Zementbomben“ genannt);

- wiederholte Unzuverlässigkeit der Patienten in der Nachkontrolle, was zur Einweisung in die geschlossene Abteilung nach Halle zur Arbeitstherapie führte;

- penible Infektionsquellenforschung.

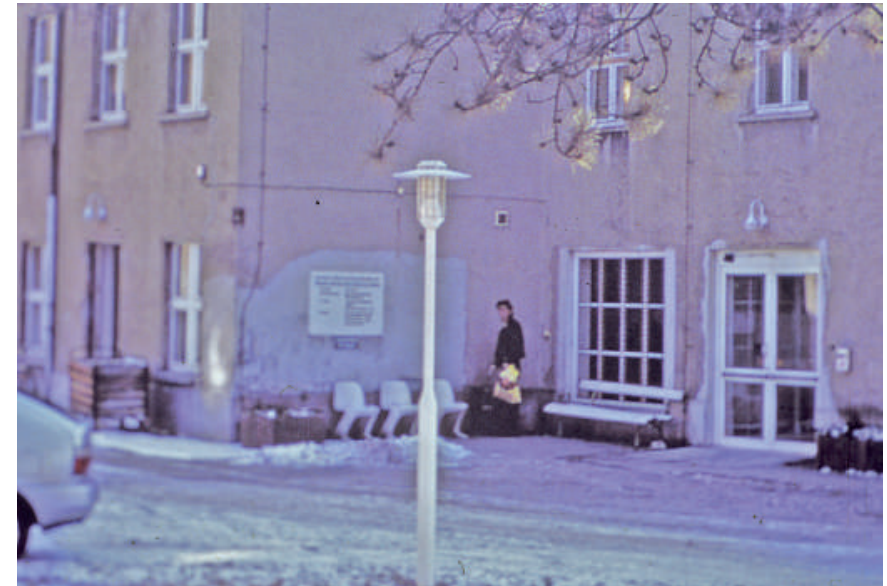

Abb. 4 Eingang zur alten Hautklinik.

1953 konnte das Gebäude Nr. 10, aus dem die Schwesternschule ausgezogen war, von der Hautabteilung belegt werden. Auf den Ruinen des früheren Kreiskrankenhauses in der Kühnauer Straße entstand die neue Hautklinik, die am 1. April 1956 als Hautklinik des Bezirkskrankenhauses Dessau eröffnet werden konnte (Abb. 4). Ab 1958 hatte die Klinik 100 Betten (55 auf der Frauenund 45 auf der Männerstation).

Von 1957 bis 1961 war Frau Dr. E. Brandt hier als Assistenzärztin und ab 1968 als Oberärztin tätig. Eine lange Reihe von Fachärzten wurde ab 1946 von Dr. Ress und seinen Nachfolgern ausgebildet. Dr. Ress hat 22 Jahre, von 1945 bis 1967, also bis zu einem Alter von 76 Jahren, die Klinik geleitet, heute unvorstellbar. Unter seiner Ägide entstanden ein allergologisches Testlabor und ein mykologisches Labor. Die Subspezialität Phlebologie fand seine besondere Förderung. Dr. Ress war Träger der Hufeland-Medaille in Gold. Er starb am 6. Januar 1975.

\section{Dr. Joachim Theune}

Dr. Ress' Nachfolger, Dr. Joachim Theune, geboren 1920, studierte 1938/39 Medizin in Posen, wurde eingezogen und verlor 1941 als Sanitätsunteroffizier durch eine Granatsplitterverwundung den rechten Arm. Nach diesem großen Opfer hatte Dr. Theune die Möglichkeit, in Prag sein Medizinstudium bis Kriegsende fortzusetzen.

1948 begann er eine Fachausbildung zum Hautarzt an der Universitätshautklinik Halle, wo er ab 1961 als 1. Oberarzt unter Prof. Grüneberg tätig war. Nachdem Dr. Ress im April 1967 ausgeschieden war, wurde Dr. Theune (Abb.5) am 15. November 1967 zum Chefarzt der Hautklinik des Bezirkskrankenhauses Dessau ernannt.

Während seiner Zeit wurde die Klinik von 100 auf 60 Betten reduziert. Dafür wurde eine Röntgenabteilung für Hautkranke in der Klinik eingerichtet und die Poliklinik musste aus der Klinik in eine gegenüberliegende Baracke umziehen. Als Oberärztin hatte er Frau Dr. Brandt, die bereits früher in Dessau gearbeitet hatte, aus Halle mitgebracht. Die Klinik hatte damals 2-3 Assistenten, und viele angehende Praktiker absolvierten hier 3 Mona- 


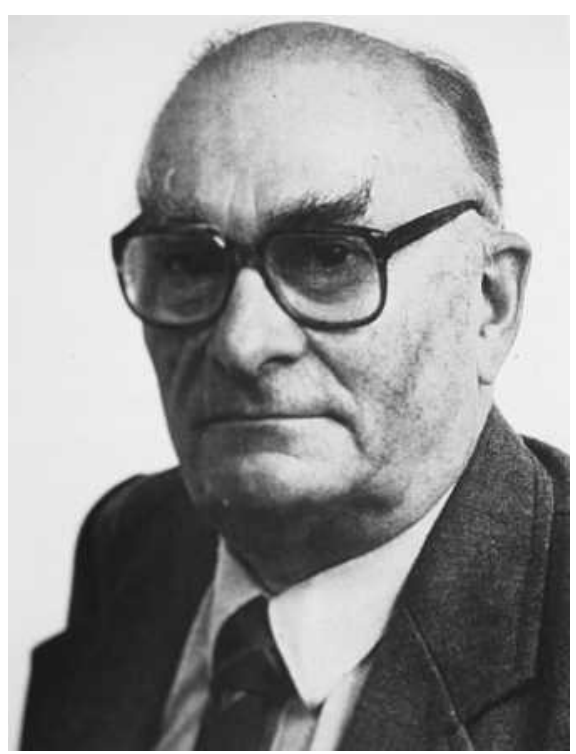

Abb. 5

Dr. Joachim Theune (1920-2006).

te Hautheilkunde. Während Dr. Theunes Zeit war es usus geworden, dass die Staatssicherheit so genannte Störer und potenzielle „Provokateure“ für 1-2 Tage an hohen DDR-Feiertagen in die Hautklinik einwies - heute eine Ungeheuerlichkeit -, die der Klinikchef still duldete oder erdulden musste. Dr. Theune starb im Februar 2006 86-jährig in Dessau.

\section{Prof. Dr. Hans-Dieter Göring}

1986 übernahm der dreifache Facharzt (Gerichtsmedizin, Dermatologie und Geschlechtskrankheiten, Immunologie) Dr. HansDieter Göring, aus Erfurt kommend, die Leitung der Klinik, um mit großem Elan deren Modernisierung voran zu treiben, so wie es unter den damaligen wirtschaftlichen Bedingungen möglich war. So entstand bald das Immunologische Zentrum, für das Dr. Göring „auf dem kleinen Dienstweg“ beim Generaldirektor von Zeiss-Jena und SED-Zentralkomitee-Mitglied Prof. Biermann ein Fluoreszenzmikroskop „locker machte“. Die Bestrahlungsgeräte zur Behandlung von Mycosis fungoides, Psoriasis und atopischer Dermatitis wurden von betroffenen Patienten, die als Ingenieure im VEB Junkalor arbeiteten, selbst konstruiert, da Importgeräte für die Dessauer Klinik unerreichbar waren, und diese Geräte „Marke Eigenbau“ funktionierten nach Dr. Görings Aussage gut. 1988 erfolgte die Ernennung von Dr. Göring zum Professor für Dermatologie.

Während der Dienstzeit von Prof. Göring erfolgte der Ausbau eines OPs aus vorher anderweitig genutzten Räumlichkeiten, die Installierung eines histologischen, mykologischen, andrologischen und immunologischen Labors. In der operativen Dermatologie führte er die schnittrandkontrollierte Exzision, die Sentinel-Lymphknoten-Detektion und -Operation sowie die Varizen und Ulkuschirurgie ein. 1998 konnte die Klinik aus der Kühnauer Straße in das neue Klinikum in Alten umziehen (Abb. 6).

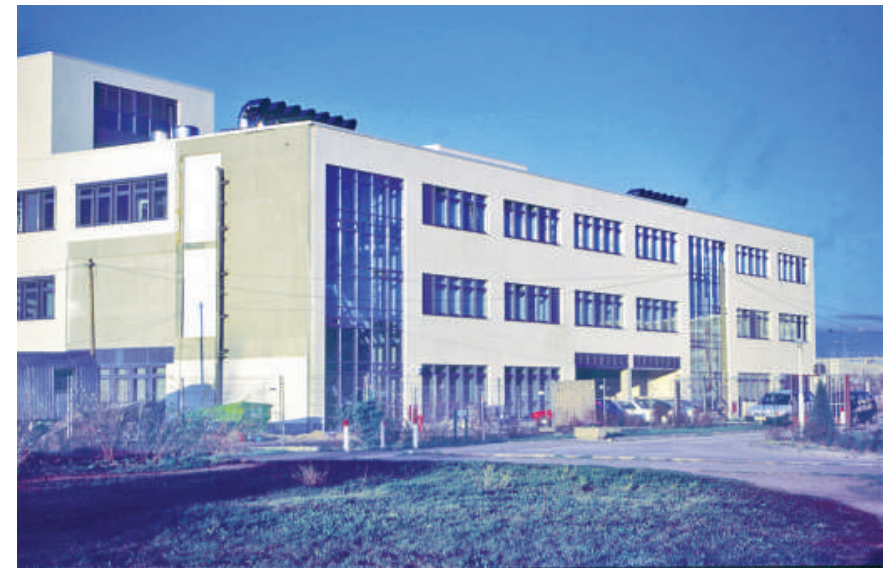

Abb. 6 Das neue Städtische Klinikum Dessau.

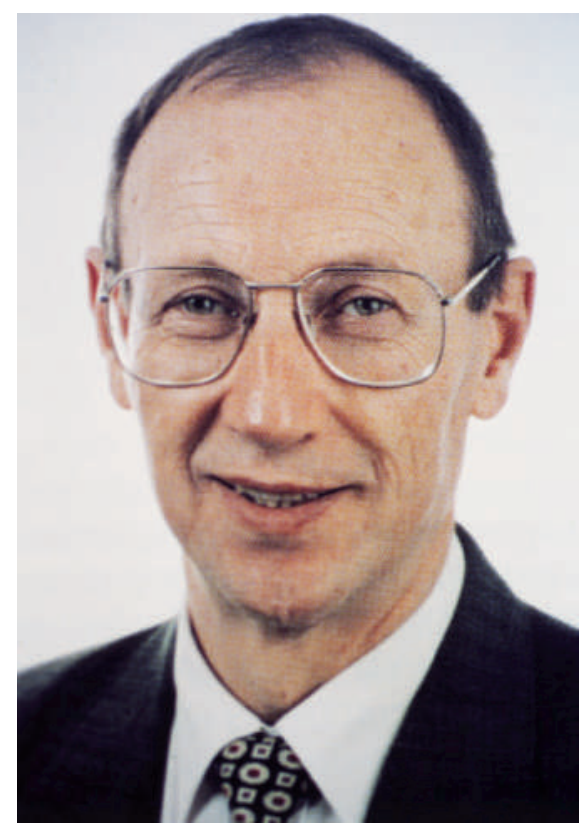

Abb. 7 Prof. Dr. Hans-Dieter Göring (geb. 1940).

Prof. Dr. Göring verfasste etwa 220 wissenschaftliche Arbeiten, hielt ca. 350 Vorträge im In- und Ausland und schrieb mehrere Bücher und Buchbeiträge, betreute mehrere Dissertationen und sogar eine Habilitation, wofür er von der Bundesärztekammer mit der Ernst-von-Bergmann-Plakette geehrt wurde. Zu seinem 65. Geburtstag erhielt Prof. Dr. Göring (Abb. 7) das Bundesverdienstkreuz und die Ehrenmitgliedschaft der Ungarischen Dermatologischen Gesellschaft. Die Ärztekammer Sachsen-Anhalt verlieh ihm ihre Ehrennadel. Kurze Zeit später ehrte ihn die Deutsche Dermatologische Gesellschaft mit der Braun-FalcoMedaille. Erst kürzlich erfolgte seine Wiederwahl zum 1. Vorsitzenden des Tumorzentrums Anhalt. Prof. Dr. Göring war 6 Jahre lang Vorsitzender der Gesellschaft für Dermatologie und Venerologie Sachsen-Anhalts. Über 15 Jahre gehörte er dem Vorstand der Deutschen Sarkoidose-Vereinigung an und war über 3 Amtsperioden Vorsitzender des Wissenschaftlichen Beirats der Deutsch-Ungarischen Dermatologischen Gesellschaft. 
Prof. Dr. Görings Nachfolger im Amt wurde 2005 Prof. Dr. Christos C. Zouboulis, 1960 in Athen geboren. Er studierte Medizin und Politische Wissenschaften an der Nationalen Universität Athen und promovierte 1988 an der Freien Universität Berlin. Seine weitere wissenschaftliche Ausbildung erfolgte an der Hautklinik der Freien Universität Berlin. 1995 habilitierte er für das Fach Dermatologie und Venerologie und wurde 2000 zum Professor der Freien Universität Berlin ernannt. Er gilt als würdiger Nachfolger Prof. Dr. Görings und wird mit dem ihm eigenen Schwung mithilfe seiner Mitarbeiter den Ruf der 40-Betten-Klinik weiter voranbringen.
Allergologie Handbuch - Grundlagen und klinische Praxis

J. Saloga, L. Klimek, R. Buhl, N. Mann, J. Knop

Stuttgart: Schattauer, 2006. 547 S., 195 meist farbige Abb., 181 Tab., Geb. 149,-€, ISBN 3-7945-1972-8

Die Herausgeber und teils auch Mitautoren Prof. Saloga, Hautklinik der Universität Mainz, Prof. Klimek, Zentrum für Rhinologie und Allergologie Wiesbaden, Prof. Buhl, III. Medizinische Klinik, Schwerpunkt Pulmologie der Universität Mainz, Prof. Mann, HNO-Klinik der Universität Mainz und Prof. Knop, Hautklinik der Universität Mainz sind die Herausgeber dieses interdisziplinären Fachbuches mit 46 Autoren, das sich trotz seiner 547 Seiten „Handbuch“ nennt. Dem Titel entsprechend werden zunächst die Grundlagen erläutert - und zwar nicht nur zu Aufbau und Funktion des Immunsystems, sondern auch zu Aufbau (Anatomie bzw. Histologie) und Funktion von Auge, Respirationstrakt, Verdauungstrakt und Haut. Weiter finden sich im Grundlagenteil Kapitel zu Neuroimmunologie, allergischen Reaktionsmechanismen, Allergenen, Genetik, Epidemiologie und psychosomatischen Aspekten allergischer Krankheiten. Es folgen - gegliedert nach Organsystemen - die Kapitel zur Klinischen Praxis der allergologischen Diagnostik, der allergologischen Krankheitsbilder, der allergologischen Therapieprinzipien und der ärztlichen Begutachtung. Letzteres behandelt u.a. die Berufskrankheiten BK 4301, allergisches Asthma bronchiale, allergische Rhinopathie, die BK 1315, Erkrankungen durch Isocyanate, die BK 4201, exogen-allergische Alveolitis und die BK 5101 Haut. Somit liegt ein umfassendes allergologisches, fächerübergreifendes Nachschlagewerk vor. Vielleicht könnte eine Wertung der Evidenz für die phytotherapeutischen Zubereitungen für Neurodermitis aufgenommen und den Medikamentenallergien etwas mehr Platz eingeräumt werden. Die Verständlichkeit des Werkes wird vor allem durch die konsequent durchgehaltene, klare Gliederung und einen eingängigen Sprachstil erreicht. Sehr gut inhaltlich und auch auflockernd sind die besonders markierten Einschübe zu „Detailwissen“ und die Anmerkungen mit Ausrufezeichen und farblicher Hervorhebung. Besonders herauszuheben sind die hervorragenden Tabellen, die vielfältigen Abbildungen und klinischen Bilder. Literatur- und Stichwortverzeichnis runden das Handbuch ab. Aktualität besitzt der Band in hohem Maße. Das Fachbuch ist zudem ein Referenzwerk und Nachschlagebuch der modernen Allergologie für Allergologen und allergologisch Interessierte aller Fachrichtungen.

Christiane Bayerl, Wiesbaden 